\title{
On the Derivation of Hawking Radiation Associated with the Formation of a Black Hole
}

\author{
Klaus Fredenhagen ${ }^{1}$ and Rudolf $\mathrm{Haag}^{2}$ \\ ${ }^{1}$ Institut für Theorie der Elementarteilchen, FU Berlin, W. Berlin, Federal Republic of Germany \\ ${ }^{2}$ II. Institut für Theoretische Physik, Universität Hamburg, Hamburg, Federal Republic of Germany
}

\begin{abstract}
We show how in gravitational collapse the Hawking radiation at large times is precisely related to a scaling limit on the sphere where the star radius crosses the Schwarzschild radius (as long as the back reaction of the radiation on the metric is neglected). For a free quantum field it can be exactly evaluated and the result agrees with Hawking's prediction. For a realistic quantum field theory no evaluation based on general principles seems possible. The outcoming radiation depends on the field theoretical model.
\end{abstract}

\section{Introduction}

Classical general relativity leads to the conclusion that very massive stars ultimately end by gravitational collapse, leading at some stage to the formation of a black hole from whose inside no signal can reach an outside observer. Furthermore, for the outside world the black hole has properties of a thermodynamic system in equilibrium ("no hair theorems", entropy) [1,2]. In an admirable paper [3] Hawking argued that the association of a temperature ${ }^{1}$

$$
T=\frac{c^{3} \hbar}{8 \pi M G}=\frac{a}{2 \pi} \hbar / c
$$

with the black hole surface could be understood by considering quantum field theory in the curved space-time given by the gravitational field of the collapsing star. The simplest model is to take a field obeying linear field equations in a spherically symmetric collapse. At early times, when the star is practically stationary, the state of the quantum field may be assumed to be the "vacuum", i.e. the lowest stationary state in the then static metric. Its development in time or, if one talks in the Heisenberg picture, the expectation values of field quantities at later times can then in principle be calculated by solving the quantum field equations once the gravitational background field is known, and this latter may

\footnotetext{
${ }^{1} M$ is the mass of the star, $a$ the acceleration at the surface of the black hole, $G$ the gravitational constant
} 
be taken from one of the classical models of gravitational collapse, e.g. the Oppenheimer-Snyder model. One has to solve then an initial value problem of a hyperbolic partial differential equation for singular functions (distributions) where the coefficients are space-time dependent and boundary conditions on the surface of the star have to be respected. Since one is interested in a tiny effect at very large times it has so far not been possible to treat the problem in this way with sufficient control of the errors. In his first paper on the subject Hawking carried through such a calculation using a geometrical optics approximation. Although there are many reasons to believe now that the resulting conclusions are essentially correct it is hard to verify to what extent this approximation is trustworthy since the light rays considered pass through regions of extremely fast changing index of refraction.

Subsequent papers have concentrated on a different approach: the discussion of a permanent, static black hole. There one has the analogy of the outside region with the Rindler universe which can be described as the wedge

$$
x^{1}>\left|x^{0}\right| ; \quad-\infty<x^{0}, x^{2}, x^{3}<\infty
$$

in Minkowski space; the boundary $x^{1}=\left|x^{0}\right|$ is a horizon for linearly accelerated observers in the wedge, following orbits of the action of a 1-parametric subgroup of the Lorentz group, the boosts in the $x^{1}$-direction

$$
x^{0}(\tau)=\rho \sinh \tau, \quad x^{1}(\tau)=\rho \cosh \tau .
$$

Without motivation from black hole physics Bisognano and Wichmann [4] had found the remarkable theorem in ordinary Minkowski space quantum field theory that the vacuum state, restricted to observation in the wedge (1.2) is a thermal state with respect to the time parameter $\tau$ of (1.3). The temperature agrees with (1.1) if the accelerations are properly compared. The parallelism between this result of [4] and the Hawking temperature was first pointed out by Sewell [5]. Unruh [6] presented arguments showing that a detector moving with linear acceleration in the vacuum state of quantum field theory in Minkowski space should respond similarly to one at rest in a medium filled by Planck radiation of this temperature.

Although these analogies are very suggestive they cannot be completely translated to the case of a spherical black hole since there we do not have the other time-like Killing vector field whose ground state defines a global vacuum. Therefore, in yet another approach, attention was focused on the local aspect of the state in the immediate vicinity of the horizon. As Fulling had first pointed out [7], the field equations and canonical commutation relations do not suffice to determine the theory. In [8] it was argued that all physically allowed states should become indistinguishable in the short distance limit of observations and that the specification of this common behaviour is an essential part of the definition of the theory ("local definiteness"). This can be done by requiring that a short distance scaling limit of the theory exists. It was then shown in $[8,9]$ that this limit defines for each point of the space-time manifold a quantum field theory in the tangent space with a distinguished state which is invariant under translations in tangent space. In the context of a classical treatment of gravitation it should be also Lorentz invariant with respect to the metric at the contraction point. It is then natural to demand that this distinguished state should be the vacuum state of the tangent 
space theory (a requirement called "local stability"). Applying this condition to the points on the horizon in the metric of a black hole one recovers the Hawking temperature in the following sense: of all thermal states (with respect to the Killing vector field of Schwarzschild time translations) only the one with temperature (1.1) satisfies the local stability condition on the horizon [8]. Another way to specify the short distance behaviour is to require that the symmetric part of the 2-point function should have the singularity structure of the "Hadamard elementary solution" of the wave equation (see [10-13] and references given therein). It has been used to derive the Hawking temperature by Kay and Wald [14] who show that if a somewhat strengthened Hadamard condition is imposed everywhere on the Kruskal extension then the only stationary quasifree states of the outside region are thermal states with temperature (1.1). As far as the strongest singularity of the 2-point function is concerned local stability and Hadamard form give the same information. Beyond this the Hadamard condition is a stronger requirement but is limited to free field theory. The scaling condition on the other hand is not quite sufficient to guarantee local definiteness (see [15] and Sect. 4 of [8]) but remains natural also in interacting theories. Related to local stability is also the postulate that the Feynman propagator should locally have an analytic continuation to imaginary times. This was the starting point in $[16,17]$ and also leads to a local temperature (1.1) as judged by the Killing vector field of Schwarzschild time translation of an outside observer.

The weakness of all arguments based on the consideration of static black holes is that they do not give direct information about the radiation due to gravitational collapse. This gap is filled here. We show that as long as the gravitational field is taken to be that produced by the matter of the star alone the expectation values of observables at any distance from the star for times $t \rightarrow \infty$ can be precisely related to a scaling limit of the state on the sphere (2-dimensional manifold), where the radius of the star crosses the Schwarzschild radius. According to the arguments in $[8,9,12,13]$ the scaling limit (the leading singularity in the short distance behaviour) is universal, i.e. the same for all allowed states in the theory. It is not affected by the previous history, by the initial conditions. The result is then indeed an asymptotically constant flow of outgoing radiation. To satisfy the (Bondi-Sachs) energy balance one has to take into account the change of the metric due to the energy of the quantum field. If, as done here, this back reaction is neglected then the infinite amount of energy radiated away in infinite time would have as its source a very small region prior in time to the crossing of the Schwarzschild radius by the surface of the star. If it is taken into account by letting the mass of the star and hence its Schwarzschild radius decrease in time then the radiation will originate from the surface of the black hole at all times after its formation and it will, of course, no longer be precisely calculable from a scaling limit. This problem will not be taken up in the present paper.

\section{Set up of the Problem and Sketch of the Main Argument}

We use coordintes $\tau, r, \vartheta, \varphi$. The spatial polar coordinates $r, \vartheta, \varphi$ are standard. The time coordinate $\tau$ is chosen so that it remains meaningful on the horizon and approaches the Schwarzschild time for $r \gg r_{o}$, where $r_{o}=2 M G / c^{2}$ is the 


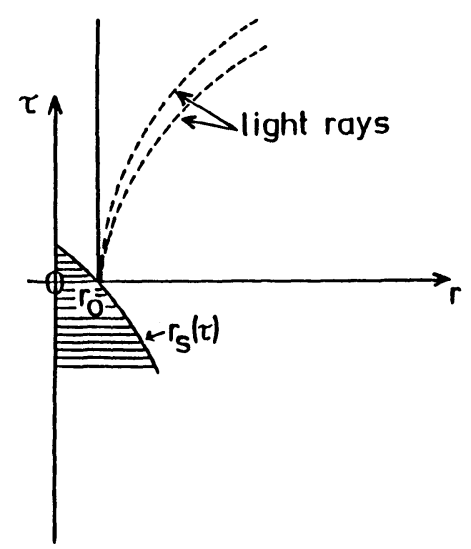

Fig. 1

Schwarzschild radius of the star mass. The boundary of the star shall be $r=r_{s}(\tau)$ (Fig. 1). The origin of the time axis is chosen so that

$$
r_{s}(0)=r_{o}
$$

i.e. the star radius crosses the Schwarzschild radius at $\tau=0$. In the outside region I:

$$
r>r_{s}(\tau) ; \quad r>r_{o}
$$

the metric is always the stationary Schwarzschild metric (Birkhoff's theorem).

In this region it is convenient to make use of the coordinates

$$
\begin{aligned}
r^{*} & =r+r_{o} \ln \left(r / r_{o}-1\right) \\
v & =t+r^{*} \\
u & =t-r^{*}
\end{aligned}
$$

(here $t$ is the Schwarzschild time) and to define our time coordinate $\tau$ by

$$
\tau=v-r=t+r^{*}-r .
$$

Far away from the horizon $r^{*} / r \rightarrow 1$. For $r \rightarrow r_{o}$ and finite $\tau$ we have $r^{*} \rightarrow-\infty$, $u \rightarrow+\infty, v$ finite. The metric is

$$
d s^{2}=-\left(1-\frac{r_{o}}{r}\right) d t^{2}+\left(1-\frac{r_{o}}{r}\right)^{-1} d r^{2}+r^{2}\left(d \vartheta^{2}+\sin ^{2} \vartheta d \varphi^{2}\right)
$$

The detailed metric inside the star matter will not be used in the following. At very early times it is nearly stationary; $r_{s}(\tau)=$ const. The precise history of the subsequent collapse will influence the radiation in the transition region but not its asymptotic behaviour for large times.

Let $\phi(x)$ be a quantum field living on this curved space-time and, for simplicity, assumed to be scalar, neutral and satisfying the covariant wave equation

$$
\begin{gathered}
\square_{g} \phi=0, \\
\square_{g}=|g|^{-1 / 2} \partial_{\mu} g^{\mu \nu}|g|^{1 / 2} \partial_{v} ; \quad|g|=-\operatorname{det} g_{\mu v} .
\end{gathered}
$$


A state is characterized by the set of expectation values

$$
W^{(n)}\left(x_{1}, \ldots, x_{n}\right)=\left\langle\phi\left(x_{1}\right) \cdots \phi\left(x_{n}\right)\right\rangle .
$$

A detector placed in some region $O$ can be simulated by $Q^{*} Q$ with

$$
Q=\int \phi(x) h(x) \sqrt{|g|} d^{4} x
$$

where $\hbar$ is a smooth function with support in $O .^{2}$ Due to the field equation $Q$ can be expressed in terms of $\phi$ and its time derivative on an arbitrarily chosen Cauchy surface. If $f$ is a $c$-number solution of

$$
\square_{g} f=0
$$

and $\Sigma_{1}, \Sigma_{2}$ are two Cauchy surfaces then

$$
\int_{\Sigma_{1}} \phi(x) \frac{\overleftrightarrow{\partial}}{\partial x^{\mu}} f(x) d \sigma^{\mu}=\int_{\Sigma_{2}} \phi(x) \frac{\overleftrightarrow{\partial}}{\partial x^{\mu}} f(x) d \sigma^{\mu},
$$

where $d \sigma^{\mu}$ denotes the surface element.

Consider now a sequence $Q_{T}(T \rightarrow \infty)$ which are time translates (with respect to the Schwarzschild time $t$ ) of a fixed $Q$ of the form (2.10) with a test function $h$ having support in a space-time region around the point $\left(0, R, \vartheta_{o}, \varphi_{o}\right)$ far away from the horizon. The function $f^{T}$ needed in (2.12) to describe $Q_{T}$ is obtained from $h$ by

$$
f^{T}(t, \underline{x})=\int d t_{o} f^{T, t_{o}}(t, \underline{x})
$$

where $f^{T, t_{o}}$ is the solution of the wave Eq. (2.11) with the initial conditions

$$
f^{T, t_{o}}\left(T+t_{o}, \underline{x}\right)=0, \quad \frac{\partial}{\partial t} f^{T, t_{o}}\left(T+t_{o}, \underline{x}\right)=h\left(t_{o}, \underline{x}\right) .
$$

The essential point is now that the space-time support of $f^{T}$ lies for $\tau \geqq 0$ entirely in the region I outside the star and outside the black hole (causal propagation). Therefore only the (stationary) Schwarzschild metric enters in the determination of $f^{T}$ for $\tau \geqq 0$. The reduction of the response rate of a far away detector at late times $T$ to properties of the quantum state at $\tau=0$ requires only the solution of the initial value problem (2.11), (2.13), (2.14) for the smooth $c$-number function $f^{T}(x)$ in the Schwarzschild metric.

Inserting the right-hand side of (2.12) with $\Sigma_{2}$ chosen as the surface $\tau=0$ into the counting rate, one gets

$$
\left\langle Q_{T}^{*} Q_{T}\right\rangle=\int_{\tau_{1}=\tau_{2}=0} W^{(2)}\left(x_{1}, x_{2}\right) \stackrel{\leftrightarrow}{D}_{1} \stackrel{\leftrightarrow}{D}_{2} f^{T}\left(x_{1}\right) f^{T}\left(x_{2}\right) d r_{1} d r_{2} r_{1}^{2} d \Omega_{1} r_{2}^{2} d \Omega_{2}
$$

with

$$
D_{i}=\left(1+\frac{r_{o}}{r_{i}}\right) \frac{\partial}{\partial \tau_{i}}-\frac{r_{o}}{r_{i}} \frac{\partial}{\partial r_{i}}
$$

\footnotetext{
${ }^{2}$ See [9], Sect. 2
} 
The asymptotic behaviour of $f^{T}$ has been studied, e.g. by Dimock and Kay [18]. From their work one can extract that, as $T \rightarrow \infty, f^{T}$ decomposes on the surface $\tau=o$ into a sum of two wave packets, one shifting with increasing $T$ towards $r \rightarrow \infty$, the other towards $r^{*} \rightarrow-\infty\left(r \rightarrow r_{o}\right)$. One may expect then that the information about the state needed in the limit $T \rightarrow \infty$ is only the 2-point function for $\tau=o$ in the immediate neighborhood of $r=r_{o}$ and for $r \rightarrow \infty$.

\section{Asymptotic Form of the Function $\boldsymbol{f}^{T}$ for $\boldsymbol{T} \rightarrow \infty$ near $\tau=0$}

We separate off the angular motion by expanding

$$
f^{T}\left(t, r^{*}, \vartheta, \varphi\right)=r^{-1} \sum_{l, m} f_{l, m}^{T}\left(t, r^{*}\right) Y_{l, m}(\vartheta, \varphi) .
$$

The wave equation (2.11) becomes then

$$
\left\{\frac{\partial^{2}}{\partial t^{2}}-\frac{\partial^{2}}{\partial r^{* 2}}+V_{l}\right\} f_{l m}^{T}=0
$$

with

$$
V_{l}(r)=\left(1-\frac{r_{o}}{r}\right)\left(\frac{l(l+1)}{r^{2}}+\frac{r_{o}}{r^{3}}\right) \text {. }
$$

Correspondingly we expand $h$. We shall discuss a single angular momentum component and suppress the indices $l, m$ where no misunderstanding is possible. $f^{T}$ is given by (2.13) and the initial conditions (physically rather the final conditions for very late $T$ ) (2.14), with $\underline{x}$ replaced by $r^{*}$. We are interested in $f^{T}\left(t, r^{*}\right)$ for $\tau$ close to zero, i.e. for $t$ close to $r-r^{*}$.

By Laplace transformation the problem may be reduced to the solution of the ordinary inhomogeneous differential equation

$$
\left(\frac{d^{2}}{d r^{* 2}}-z^{2}-V_{l}\right) \tilde{f}\left(z, r^{*}\right)=F\left(z, r^{*}\right) \equiv \int d t_{o} e^{z t_{o}} h\left(t_{o}, r^{*}\right)
$$

together with the boundary conditions

$$
\tilde{f}\left(z, r^{*}\right) \rightarrow 0 \text { for } r^{*} \rightarrow \pm \infty,
$$

where $f^{T}\left(t, r^{*}\right)$ is given by

$$
f^{T}\left(t, r^{*}\right)=(2 \pi i)^{-1} \int_{c-i \infty}^{c+i \infty} d z \tilde{f}\left(z, r^{*}\right) e^{-z(t-T)}, \quad c>0
$$

for values of $t<T+t_{1}$ with $t_{1}=\inf \left\{t_{o} \mid\left(t_{o}, r^{*}\right) \in \operatorname{supph}\right\}$.

Let us denote by $G_{+}, G_{-}$the solutions of the homogeneous equation

$$
\left(\frac{d^{2}}{d r^{* 2}}-z^{2}-V_{l}\right) G=0
$$

with respective boundary conditions

$$
G_{-} e^{-z r^{*} \rightarrow 1} \text { for } r^{*} \rightarrow-\infty, \quad G_{+} e^{z r^{*} \rightarrow 1} \text { for } r^{*} \rightarrow+\infty .
$$


$G_{+}, G_{-}$are uniquely determined for $\operatorname{Re} z \geqq 0, z \neq 0$. They are analytic functions of $z$ for $\operatorname{Re} z>0$. Due to the fact that $V_{l}$ decreases exponentially like $e^{\boldsymbol{r}^{*} / r^{\boldsymbol{o}}}$ for $r^{*} \rightarrow-\infty$ the analyticity of $G_{-}\left(z, r^{*}\right)$ in $z$ extends into the left half plane till $2 \operatorname{Re} z>-r_{o}$, and $G_{-}$is a smooth function of $r^{*}$. This can be seen if one writes down the explicit solution

$$
\begin{aligned}
G_{-}\left(z, r^{*}\right)= & e^{z r^{*}}+\sum_{n=1}^{\infty} \int_{-\infty}^{r^{*}} d y_{1} \int_{-\infty}^{y_{1}} d y_{2} \ldots \int_{-\infty}^{y_{n-1}} d y_{n} \frac{\sinh z\left(r^{*}-y_{1}\right)}{z} \ldots \frac{\sinh z\left(y_{n-1}-y_{n}\right)}{z} \\
& \cdot e^{y_{n} z} V\left(y_{n}\right) \cdots V\left(y_{1}\right)
\end{aligned}
$$

which converges for all $z$ in the mentioned region of the complex plane. In the case of $G_{+}$the long range tail of $V_{l} \sim\left(l(l+1) / r^{* 2}\right)$ for $r^{*} \rightarrow \infty$ allows only a weaker statement. The expansion corresponding to (3.9) gives a series which still converges on the imaginary axis with the exception of $z=0$. The tail of the potential differs from the dominating but exactly solvable $1 / r^{* 2}$-decreases by $\Delta V_{l}$ for which $r^{*} . \Delta V_{l}$ is absolutely integrable for $r^{*} \rightarrow \infty$. This implies that $G_{+}$behaves like $z^{-l}$ for $z \rightarrow 0$. One finds that $z^{l} G_{+}$is continuous on the imaginary axis. In fact, using the techniques developed in [19] one proves that, apart from the point $z=o, z^{l} G_{+}$is analytic (hence smooth) on the imaginary axis. In terms of $G_{-}$and $G_{+}, \tilde{f}$ is given by

$$
\tilde{f}\left(z, r^{*}\right)=\frac{z^{l}}{\delta(z)}\left\{G_{+}\left(z, r^{*}\right) \int_{-\infty}^{r^{*}} d r^{*^{\prime}} G_{-}\left(z, r^{*^{\prime}}\right) F\left(z, r^{*^{\prime}}\right)+G_{-}\left(z, r^{*}\right) \int_{r^{*}}^{\infty} d r^{*^{\prime}} G_{+}\left(z, r^{*^{\prime}}\right) F\left(z, r^{*^{\prime}}\right)\right\} \text {. }
$$

Here $\delta(z)$ is the Wronskian between $G_{-}$and $z^{l} G_{+}$,

$$
\delta(z)=z^{l}\left(G_{+} \frac{\partial G_{-}}{\partial r^{*}}-\frac{\partial G_{+}}{\partial r^{*}} G_{-}\right)
$$

Using the positivity of $V\left(r^{*}\right)$ one shows that $\delta(z)$ cannot vanish on the imaginary axis.

On the imaginary $z$-axis the function $F$, being the Fourier transform of the function $h$ describing the detector, is smooth. We may choose $F$ so that it as well as some of its derivatives vanish at $z=o$ if we are not interested in detecting quanta of zero energy. Then $\tilde{f}\left(i \omega, r^{*}\right)$ is (multiply) differentiable for all $\omega$. It vanishes fast for $|\omega| \rightarrow \infty$ if $F\left(i \omega, r^{*}\right)$ does. The function $f^{T}$ as well as its derivatives will tend to zero uniformly in $r^{*}$ within any fixed finite interval of $r^{*}$ as $T-t \rightarrow \infty$.

For $r^{*}$ to the left (respectively to the right) of the support of $F, f^{T}\left(t, r^{*}\right)$ has the form

$$
f^{T}\left(t, r^{*}\right)=(2 \pi)^{-1} \int d \omega \frac{(i \omega)^{l}}{\delta(i \omega)} G_{\mp}\left(i \omega, r^{*}\right) c_{ \pm}(i \omega) e^{-i \omega(t-T)}
$$

where $c_{ \pm}$depends on the initial conditions

$$
c_{ \pm}(i \omega)=\int_{-\infty}^{+\infty} d r^{*} G_{ \pm}\left(i \omega, r^{*}\right) F\left(i \omega, r^{*}\right)
$$

The leading term in $G_{+}$is a spherical Hankel function describing an outgoing 
wave with angular momentum $l$, the leading term in $G_{-}$is a leftgoing wave in a 2-dimensional space-time. For $T-t \rightarrow \infty$ we may then decompose $f^{T}$ into the three parts

$$
f^{T}=f_{-}^{T}+f_{+}^{T}+\Delta^{T},
$$

where $f_{ \pm}^{T}$ are given by (3.12) with $G_{ \pm}$replaced by their leading terms. The function $\Delta^{T}\left(t, r^{*}\right)$ is a smooth function which tends together with its derivatives uniformly to zero as $T \rightarrow \infty$ for all $\left(t, r^{*}\right)$ in a neighborhood of the surface $\tau=0$ because $f^{T}$ tends to zero in any finite interval of $r^{*}, f_{-}^{T}$ tends to zero in any half infinite interval $r^{*}>a$ and $f_{+}^{T}$ tends to zero in any half infinite interval $r^{*}<b$.

For $f_{-}^{T}$ we have

$$
f_{-}^{T}\left(t, r^{*}\right)=\phi\left(r^{*}-t+T\right)
$$

with

$$
\begin{gathered}
\phi(u)=(2 \pi)^{-1} \int d \omega a(\omega) e^{i \omega u} \\
a(\omega)=\frac{(i \omega)^{l}}{\delta(i \omega)} c_{+}(i \omega),
\end{gathered}
$$

$f_{-}^{T}$ is a wave packet accumulating at $\tau=0$ on the horizon. In the variables $\tau, r$ it is of the form

$$
f_{-}^{T}(\tau, r)=\psi\left(\frac{\xi}{\lambda} e^{-\left(\tau / 2 r_{o}\right)}\right)
$$

with

$$
\xi=\frac{r-r_{o}}{r_{o}}, \lambda=e^{-\left(T / 2 r_{o}\right)}, \quad \psi\left(e^{y / 2 r_{o}}\right)=\phi(y) .
$$

We have neglected irrelevant factors tending to 1 for $r \rightarrow r_{o}$.

\section{Asymptotic Counting Rate and Short Distance Behaviour of the State}

To evaluate the counting rate in the limit $T \rightarrow \infty$ we need some qualitative information about $W^{(2)}$ in the neighborhood of the spacelike surface $\tau=0$. We assume that for spacelike separation $W^{(2)}$ is together with its derivatives a smooth function which is bounded at infinity, that it approaches the ground-state-2-pointfunction for $r_{1}^{*}, r_{2}^{*} \rightarrow \infty$ and that its short distance behaviour is of the form $[8,9]$

$$
W^{(2)}\left(x_{1}, x_{2}\right)=(2 \pi)^{-2} \sigma_{\varepsilon}^{-1}+\hat{w}^{(2)},
$$

where $\sigma_{\varepsilon}$ is the square of the geodesic distance between $x_{1}$ and $x_{2}$ with $\tau_{1}$ replaced by $\tau_{1}-i \varepsilon, \tau_{2}$ by $\tau_{2}+i \varepsilon$ and the limit $\varepsilon \downarrow 0$ is understood. $\hat{w}^{(2)}$ is less singular, i.e. $\sigma \hat{w}^{(2)}$ and $\left(\partial / \partial x_{1}^{\mu}\right)\left(\partial / \partial x_{2}^{\nu}\right) \hat{w}^{(2)} /\left(\partial / \partial x_{1}^{\mu}\right)\left(\partial / \partial x_{2}^{\mu}\right) \sigma_{\varepsilon}^{-1}$ are continuous and vanish on the light cone $\sigma=0$. This is true if $W^{(2)}$ has the so-called Hadamard form. We expect that the assumed properties of $W^{(2)}$ can be derived from the fact that $W^{(2)}$ at earlier times is the 2-point function of a ground state in a static background by an extension of the results in $[12,13]$. 
The counting rate (2.17) is bilinear in $f^{T}$. We insert the decomposition (3.14). The diagonal terms with $f_{+}^{T}$ and $f_{-}^{T}$ are bounded, the term with $\Delta^{T}$ vanishes in the limit $T \rightarrow \infty$. Thus by positivity of $W^{(2)}$ all terms involving $\Delta^{T}$ tend to zero. The cross term between $f_{+}^{T}$ and $f_{-}^{T}$ involves only integration over spacelike separated points, up to contributions which vanish in the limit $T \rightarrow \infty$. So, using the assumed behaviour of $W^{(2)}$ at spacelike separation, we estimate this cross term by

$$
\text { const. } \int\left|f_{+}^{T}\right| \int\left|f_{-}^{T}\right| \leqq \text { const. } T^{2} e^{-\left(T / 2 r_{0}\right)} \rightarrow 0 .
$$

The contribution where both factors $f^{T}$ are replaced by $f_{+}^{T}$ is not affected by the collapse of the star. It approaches the response of the detector in the globally static ground state of the quantum field in the metric of a stationary extended star and is zero for a passive detector.

We are then left with the part of (2.17) where both functions $f^{T}$ are replaced by $f_{-}^{T}$. This is governed by the short distance behaviour of the state near the horizon at $\tau=0$.

To simplify the expression (2.17) we note that

$$
D u=-\frac{\partial u}{\partial r}, \quad D v=\frac{\partial v}{\partial r} .
$$

Since $f_{-}^{T}$ is a function of $u$ alone we have $D f=-(\partial f / \partial r)$. By partial integration and replacement of $r$ by $r_{o}$ in the uncritical positions we get for the horizon contribution

$$
\lim _{T \rightarrow \infty}\left\langle Q_{T}^{*} Q_{T}\right\rangle=\lim _{\lambda \rightarrow 0} 2 \pi r_{o}^{6} \sum_{l, m, l^{\prime}, m^{\prime}} \int W_{l, m, l^{\prime}, m^{\prime}}^{\prime \prime}\left(\xi_{1}, \xi_{2}\right) \psi_{l m}\left(\frac{\xi_{1}}{\lambda}\right) \psi_{l^{\prime}, m^{\prime}}\left(\frac{\xi_{2}}{\lambda}\right) d \xi_{1} d \xi_{2},
$$

where $\xi_{i}=\left(r_{i}-r_{o}\right) / r_{o}$ and

$$
\begin{aligned}
& W_{l, m, l^{\prime}, m^{\prime}}^{\prime \prime}\left(\xi_{1}, \xi_{2}\right)=\int W^{\prime \prime}\left(r_{o}\left(1+\xi_{1}\right), r_{o}\left(1+\xi_{2}\right), \vartheta_{1}, \varphi_{1}, \vartheta_{2}, \varphi_{2}\right) \\
& \cdot \overline{Y_{l m}\left(\vartheta_{1}, \varphi_{1}\right)} Y_{l^{\prime} m^{\prime}}\left(\vartheta_{2}, \varphi_{2}\right) d \Omega_{1} d \Omega_{2}, \\
& W^{\prime \prime}\left(r_{1}, r_{2}, \vartheta_{1}, \varphi_{1}, \vartheta_{2}, \varphi_{2}\right)=\left.\hat{D}_{1} \hat{D}_{2} W^{(2)}\left(x_{1}, x_{2}\right)\right|_{\tau_{1}=\tau_{2}=0} \text {, } \\
& \hat{D}_{i}=2\left(\frac{\partial}{\partial \tau_{i}}-\frac{\partial}{\partial r_{i}}\right) \text {. }
\end{aligned}
$$

For small distances one has

$$
\begin{gathered}
\sigma_{\varepsilon}=r_{o}^{2}\left(K_{\varepsilon}^{2}+\vartheta_{12}^{2}\right), \\
K_{\varepsilon}^{2}=\left(\xi_{1}-\xi_{2}+i \varepsilon\right) K_{v}, \quad K_{v}=\left(\tau_{1}-\tau_{2}+r_{1}-r_{2}-i \varepsilon\right) \frac{2 e^{1 / 2}}{r_{o}},
\end{gathered}
$$

where $\vartheta_{12}$ is the angle between $\left(\vartheta_{1}, \varphi_{1}\right)$ and $\left(\vartheta_{2}, \varphi_{2}\right)$. Inserting $(4.1)$ into (4.6) yields

$$
W^{\prime \prime}=\left[(2 \pi)^{-2}\left(\frac{2 \hat{D}_{1} \sigma \hat{D}_{2} \sigma}{\sigma_{\varepsilon}^{3}}-\frac{\hat{D}_{1} \hat{D}_{2} \sigma}{\sigma_{\varepsilon}^{2}}\right)+\hat{D}_{1} \hat{D}_{2} \hat{W}^{(2)}\right]_{\mid \tau_{1}=\tau_{2}=0}
$$


Using (4.8) we get for $\tau_{1}=\tau_{2}=0, \hat{D}_{1,2} \sigma= \pm K_{v}, \hat{D}_{1} \hat{D}_{2} \sigma=0$ and

$$
\lim _{\lambda \rightarrow 0} \lambda^{2} \frac{\hat{D}_{1} \sigma \hat{D}_{2} \sigma}{\sigma_{\varepsilon}^{3}}\left(\lambda \xi_{1}, \lambda \xi_{2}, \vartheta_{12}\right)=-r_{0}^{-3}\left(\xi_{1}-\xi_{2}+i \varepsilon\right)^{-2} \frac{\frac{1}{2} \delta\left(\vartheta_{12}\right)}{\sin \vartheta_{12}} .
$$

The contribution of $\hat{W}^{(2)}$ vanishes in the limit $\lambda \rightarrow 0$. So we find

$$
\lim _{T \rightarrow \infty}\left\langle Q_{T}^{*} Q_{T}\right\rangle=\text { const. } \sum_{l m} \int d \xi_{1} d \xi_{2}\left(\xi_{1}-\xi_{2}+i \varepsilon\right)^{-2} \overline{\psi_{l m}\left(\xi_{1}\right)} \psi_{l m}\left(\xi_{2}\right) .
$$

To bring this into a more readily interpretable form we go back to (3.15-19). Up to a normalization constant the right-hand side of $(4.12)$ is

$$
\sum_{l m} \int\left[\sinh \left(\frac{y_{1}-y_{2}}{4 r_{o}}+i \varepsilon\right)\right]^{-2} \overline{\phi_{l m}\left(y_{1}\right)} \phi_{l m}\left(y_{2}\right) d y_{1} d y_{2}=\sum_{l m} \int \frac{\left|a_{l m}(\omega)\right|^{2} \omega d \omega}{1-e^{-4 \pi r_{o} \omega}} .
$$

We note that

$$
\frac{(i \omega)^{l}}{\delta(i \omega)}=\frac{D_{l}(\omega)}{2 i \omega}
$$

where $D_{l}$ is the transmission amplitude through the potential barrier (3.3). If we replace in (3.13) $G_{+}$by it asymptotic form $e^{i \omega r^{*}}$ we get

$$
a_{l m}(\omega)=D_{l}(\omega) \frac{\tilde{h}_{l m}(\omega,-\omega)}{2 i \omega}
$$

Thus we get finally $\left(\beta=4 \pi r_{o}\right)$

$$
\left.\lim _{T \rightarrow \infty}\left\langle Q_{T}^{*} Q_{T}\right)\right\rangle=\text { const. } \sum_{l, m} \int_{-\infty}^{+\infty} d \omega\left|D_{l}(\omega)\right|^{2} \frac{\left|\tilde{h}_{l m}(\omega,-\omega)\right|^{2}}{\omega\left(1-e^{-\beta \omega}\right)} .
$$

Equation (4.16) shows that the asymptotic counting rate is the one produced by an outgoing radiation with temperature $\left(4 \pi r_{o}\right)^{-1}$ modified by the barrier penetration effect.

\section{Summary and Conclusions}

The result (4.16) is just a corroboration of Hawking's original prediction. Apart from the effort spent in making the derivation watertight in the case of a free quantum field the main interest of the preceding discussion lies in the clarification of the essential steps which lead to this result. Namely the relation between the restriction of the state to an observation region far away from the black hole at very large times and the short distance behavior of the state on the sphere $r=r_{0}$ at the instant when the star radius crosses the Schwarzschild radius and the claim that the short distance behavior is universal for all allowed states in the theory. The surprising aspect of the result is that the radiation caused by the changing metric field of the collapsing star approaches a steady outgoing flow for large times which means a drastic violation of energy conservation for the total system if one neglects the back reaction of the (quantum) radiation of the causal structure of space-time. Therefore a full understanding of the phenomenum including a 
self-consistent description of the causal structure needs some elements of a quantum theory of gravity. In spite of many efforts they have not yet been elucidated in a satisfactory manner. To turn it around: this aspect provides an important consistency condition in the quest for a symbiosis of general relativity and quantum physics.

The other interesting aspect is the degree of universality (model independence). While it appears that the Bekenstein entropy and the Hawking temperature are completely model independent these quantities do not have a direct observational significance since they concern the "naked" black hole relative to the vector field of time translations which is distinguished as an asymptotic symmetry far away in the outside region ${ }^{3}$. The radiation which is transmitted to the outside depends significantly on the quantum field theory model. In the case of a free quantum field the modification of thermal radiation is simply determined by the transmission coefficients $\left|D_{l}(\omega)\right|^{2}$ through the potential barrier (3.3). If we envisage instead a realistic quantum field theory, say the standard model of elementary particle physics, we can adapt some of the preceding arguments. Again we can expect that (as long as the back reaction is neglected and the theory is asymptotically free in the high energy limit) the dynamical equations define a mapping of observables far away at time $T \rightarrow \infty$ into the tangent space algebras of the points on the sphere $\tau=0, r=r_{o}$. Again all physical states will coincide to give the same expectation functional on the tangent space algebras. We also know some properties of the mapping, in particular that time translations of the asymptotic observables correspond to dilations of their images in the tangent space algebra. However, the computation of the emitted radiation demands a detailed knowledge of the mapping and this is a highly nontrivial problem in quantum field theory. In QCD for example the scaling limit of a state gives the ground state for a theory of free quarks and gluons in the tangent space. The operator whose expectation value in this state we need to evaluate is determined in principle from the operator representing a detector of hadrons, placed well outside the black hole, via the dynamical equations of QCD in the gravitational background field provided by the star. For a first orientation one may neglect the gravitational field at distances beyond, say, $R=10 r_{o}$, and take an idealized detector appropriate to the Minkowski space theory placed at this distance $R$ but at a very late time $T$ so that the backward light rays from the region of placement cut the hyperplane $\tau=0$ very close to the Schwarzschild radius. The dynamical law in the presence of the gravitational field may be regarded in a vicinity of each point as the dynamical law of ordinary QCD in the reference frame of the local 4-bein. For the observable of interest one gets as the time approaches $\tau=0$ into the short distance regime of QCD, i.e. one approaches the asymptotically free limit. The transition from $\tau=T$ to $\tau=0$ (which in the simple model treated in this paper is described as the penetration of a potential barrier) poses now a tough problem even if one is content with a qualitative treatment. In the language of present day elementary particle physics

\footnotetext{
${ }^{3}$ As long as the back reaction is neglected it is a symmetry in the whole outside region, but realistically it is distinguished only at large distance
} 
it involves the fragmentation of hadrons due to a gradual fading of the effective interaction between quarks and gluons.

\section{References}

1. Hawking, S. W., Ellis, G. F. R.: The large scale structure of space-time, Cambridge: Cambridge University Press 1980

2. Bekenstein, J. D.: Phys. Rev. D7, 2333 (1973)

3. Hawking, S. W.: Commun. Math. Phys. 43, 199 (1975)

4. Bisognano, J. J. Wichmann, E. H.: J. Math. Phys. 17, 303 (1976)

5. Sewell, G. L.: Phys. Lett. 79A, 23 (1980)

6. Unruh, W. G.: Phys. Rev. D14, 870 (1976)

7. Fulling, S. A.: Phys. Rev. D7, 2850 (1973)

8. Haag, R., Narnhofer, H., Stein, U.: Commun. Math. Phys. 94, 219 (1984)

9. Fredenhagen, K., Haag, R.: Commun. Math. Phys. 108, 91 (1987)

10. Adler, S., Liebermann, J., Ng, Y. J.: Ann. Phys. 106, 279 (1978); Adler, S., Liebermann, J.: Ann. Phys. 113, 294 (1977)

11. Wald, R. M.: Commun. Math. Phys. 54, 1 (1977); Phys. Rev. D17, 1477 (1978)

12. Fulling, S. A., Sweeny, M., Wald, R. M.: Commun. Math. Phys. 63, 257 (1981)

13. Fulling, S. A., Narcowich, F. J., Wald, R. M.: Ann. Phys. (N.Y.) 136, 243 (1981)

14. Kay, B. S., Wald, R. M.: Proceedings of the $\mathrm{XV}^{\text {th }}$ international conference on differential geometric methods in theoretical physics (Clausthal 1986). Doebner, H. D., Henning, J. D. (eds.). Singapore: World Scientific 1987; Kay, B. S., Wald, R. M.: Theorems on the uniqueness and thermal properties of stationary, nonsingular, quasi-free states on spacetime with a bifurcate killing horizon (Preprint 1988)

15. Bernard, D.: Phys. Rev. D33, 3581 (1986)

16. Hartle, J. R., Hawking, S. W.: Phys. Rev. D13, 2188 (1976)

17. Gibbons, E. W., Hawking, S. W.: Phys. Rev. D15, 2738 (1977)

18. Dimock, J., Kay, B. S.: Ann. Phys. (N.Y.) 175, 366 (1987)

19. De Alfaro, V., Regge, T.: Potential scattering. Amsterdam: North-Holland 1965

Communicated by A. Jaffe

Received April 25, 1989; in revised form July 21, 1989 